7-20-1994

\title{
Rainbow Scattering by a Coated Sphere
}

James A. Lock

Cleveland State University, j.lock@csuohio.edu

J. Michael Jamison

Chih-Yang Lin

Follow this and additional works at: https://engagedscholarship.csuohio.edu/sciphysics_facpub

Part of the Physics Commons

How does access to this work benefit you? Let us know!

\section{Publisher's Statement}

This paper was published in Applied Optics and is made available as an electronic reprint with the permission of OSA. The paper can be found at the following URL on the OSA website: http://www.opticsinfobase.org/ao/abstract.cfm?URI=ao-33-21-4677. Systematic or multiple reproduction or distribution to multiple locations via electronic or other means is prohibited and is subject to penalties under law.

\section{Original Citation}

Lock, James A., J. Michael Jamison, and Chih-Yang Lin. "Rainbow Scattering by a Coated Sphere." Applied Optics 33 (1994): 4677-4690.

\section{Repository Citation}

Lock, James A.; Jamison, J. Michael; and Lin, Chih-Yang, "Rainbow Scattering by a Coated Sphere" (1994). Physics Faculty Publications. 55.

https://engagedscholarship.csuohio.edu/sciphysics_facpub/55

This Article is brought to you for free and open access by the Physics Department at EngagedScholarship@CSU. It has been accepted for inclusion in Physics Faculty Publications by an authorized administrator of EngagedScholarship@CSU. For more information, please contact library.es@csuohio.edu. 


\title{
Rainbow scattering by a coated sphere
}

\author{
James A. Lock, J. Michael Jamison, and Chih-Yang Lin
}

\begin{abstract}
We examine the behavior of the first-order rainbow for a coated sphere by using both ray theory and Aden-Kerker wave theory as the radius of the core $a_{12}$ and the thickness of the coating $\delta$ are varied. As the ratio $\delta / a_{12}$ increases from $10^{-4}$ to 0.33 , we find three classes of rainbow phenomena that cannot occur for a homogeneous-sphere rainbow. For $\delta / a_{12} \leq 10^{-3}$, the rainbow intensity is an oscillatory function of the coating thickness, for $\delta / a_{12} \approx 10^{-2}$, the first-order rainbow breaks into a pair of twin rainbows, and for $\delta / a_{12} \approx 0.33$, various rainbow-extinction transitions occur. Each of these effects is analyzed, and their physical interpretations are given. A Debye series decomposition of coated-sphere partial-wave scattering amplitudes is also performed and aids in the analysis.
\end{abstract}

\section{Introduction}

In the scattering of a family of parallel light rays by a homogeneous spherical particle, the scattering angle $\theta$ of the rays that make one internal reflection within the particle possesses a relative minimum when considered as a function of the ray impact parameter. The first-order rainbow occurs at this relative minimum scattering angle. ${ }^{1,2}$ In wave theory, the form of the rainbow changes only superficially as the wavelength of the incident light, $\lambda$, the radius of the particle, $a$, and the particle's refractive index $n$ are varied. As $a$ increases or $\lambda$ decreases, the rainbow intensity slowly increases above the geometric optics background, the angular size of the supernumerary structure on the illuminated side of the rainbow decreases, and the scattering angle of the peak rainbow intensity approaches the Descartes rainbow angle $\theta^{R}$ of ray theory. As the refractive index of the particle increases, the Descartes rainbow angle increases. For $n=2$, the rainbow occurs at $\theta^{R}=180^{\circ}$ and is known as a rainbow-enhanced glory. ${ }^{3-6}$ For $n>2$, the scattering angle of the rays that make one internal reflection no longer possesses a relative minimum, and the first-order rainbow does not occur.

Although it has been over forty years since the problem of electromagnetic-wave scattering by a

J. A. Lock is with the Department of Physics, Cleveland State University, Cleveland, Ohio 44115; J. M. Jamison is with ADF, Inc. Cleveland, Ohio 44135; and C.-Y. Lin is with the Department of Computer Science, Pennsylvania State University at Shippensburg, Shippensburg, Pennsylvania 17257.

Received 8 September 1993; revised manuscript received 12 November 1993.

0003-6935/94/214677-14\$06.00/0.

(c) 1994 Optical Society of America. coated sphere was solved by Aden and Kerker, ${ }^{7}$ to our knowledge, a systematic study of the properties of coated-sphere rainbows has not been undertaken. It is the purpose of this paper to examine the behavior of the first-order rainbow for a coated sphere when the thickness of the coating $\delta$ is varied with respect to the radius $a_{12}$ of the core particle. For this variation, we find three classes of rainbow phenomena that have no counterparts for homogeneous-sphere rainbows. First, when $\delta / a_{12} \leq 10^{-3}$, the coating acts as a thin film on the surface of the core. As a result, as $\delta / a_{12}$ increases, the rainbow intensity alternately increases and decreases because of the constructive and the destructive interferences of the two Descartes rainbow rays that internally reflect off the core-coating interface and the coating-air interface. Second, when $\delta / a_{12} \approx 10^{-2}$, the two Descartes rainbow rays are deflected by different amounts within the coated sphere. As a result, the first-order rainbow breaks into twin rainbows at slightly differing scattering angles. Finally, when $\delta / a_{12} \approx 0.3$ for certain combinations of the core and the coating refractive indices, there is a transition at which the first-order rainbow is extinguished. We examine each of these three rainbow phenomena by using both ray theory and the Aden-Kerker solution of the electromagnetic-wave scattering problem.

The body of this paper proceeds as follows. In Section 2 we examine the thin-film situation and the resulting rainbow-intensity oscillations. In Section 3 we examine the twin-rainbow situation. Both of these results demonstrate that for large particles with $\left(2 \pi a_{12}\right) / \lambda \gtrsim 3000$, ray theory becomes a good approximation to the results of the Aden-Kerker wave theory. For a homogeneous sphere, the link between ray theory and Mie wave theory is provided 
by the Debye-series expansion of the Mie partial-wave scattering amplitudes. ${ }^{8-11}$ To provide a similar link between ray theory and wave theory for the coatedsphere problem, in Section 4 we carry out a Debyeseries decomposition of the Aden-Kerker partialwave scattering amplitudes. For completeness, we also give the Debye-series decomposition of the internal partial-wave amplitudes both in the core and the coating, and we relate these internal Debye amplitudes to arbitrary beam-scattering theory. ${ }^{12,13}$ In Section 5 we employ the Debye-series results to examine rainbow-extinction transitions for two different classes of light ray trajectories through the coated spheres. Finally, in Section 6 we briefly describe experimental observations of rainbow glare spots on a coated sphere.

\section{Coated Sphere as a Thin-Film Problem}

Consider a plane wave of field strength $E_{0}$, wavelength $\lambda$, traveling in the $z$ direction, and polarized in the $x$ direction incident upon a coated sphere that comprises a core (region 1), surrounded by a coating (region 2), and surrounded by air (region 3). The radius and the refractive index of the core are $a_{12}$ and $n_{1}$, respectively. The thickness of the coating and its refractive index are $\delta$ and $n_{2}$, respectively. The refractive index of air is $n_{3}$. The radius of the composite particle is then

$$
a_{23}=a_{12}+\delta .
$$

The geometry is shown in Fig. 1(a). The size parameters associated with the core and the composite radii are

$$
\begin{aligned}
& x_{12}=\frac{2 \pi a_{12}}{\lambda}, \\
& x_{23}=\frac{2 \pi a_{23}}{\lambda},
\end{aligned}
$$

respectively.

In this section only, the scattering angle is denoted by a lowercase $\theta$.

The Aden-Kerker expression for the far-field intensity of the light scattered by the coated sphere is ${ }^{7,14,15}$

$$
I(r, \theta, \phi)=\frac{E_{0}{ }^{2}}{2 \mu_{0} c} \frac{1}{k^{2} r^{2}}\left[\left|S_{2}(\theta)\right|^{2} \cos ^{2} \phi+\left|S_{1}(\theta)\right|^{2} \sin ^{2} \phi\right],
$$

where

$$
k=\frac{2 \pi}{\lambda}
$$

is the wave number of the incident plane wave, and

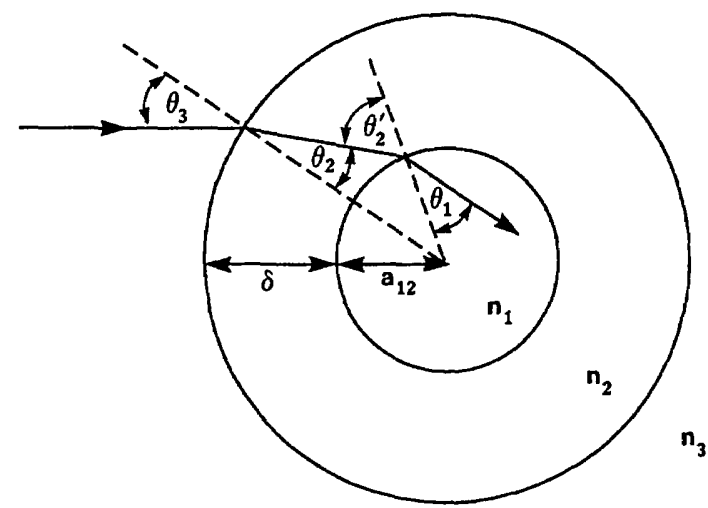

(a)

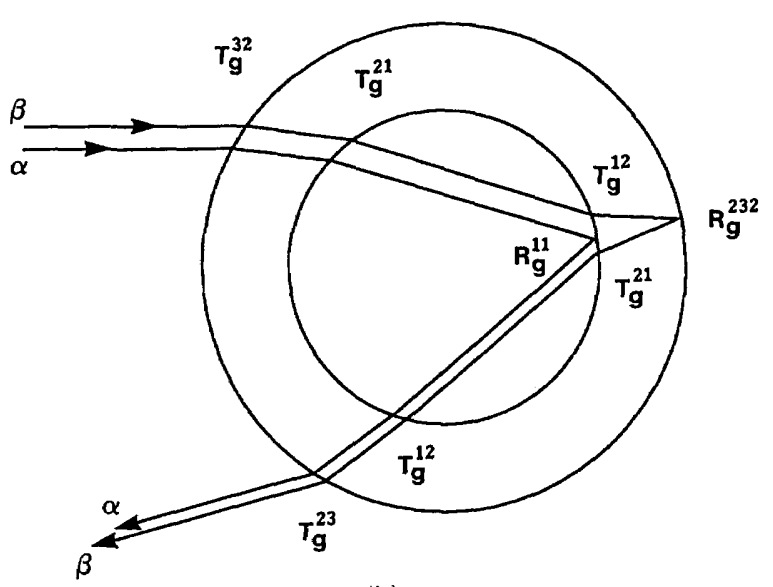

(b)

Fig. 1. (a) Geometry of a coated sphere and the trajectory of a geometric light ray through it. (b) Two geometric rays $\alpha$ and $\beta$ that dominate the first-order rainbow for a coated sphere. The flat interface Fresnel coefficients for the various transmissions and internal reflections are also indicated.

the scattering amplitudes $S_{1}(\theta)$ and $S_{2}(\theta)$ are given by

$$
\begin{aligned}
& S_{1}(\theta)=\sum_{l=1}^{\infty} \frac{2 l+1}{l(l+1)}\left[a_{l} \pi_{l}(\theta)+b_{l} \tau_{l}(\theta)\right], \\
& S_{2}(\theta)=\sum_{l=1}^{\infty} \frac{2 l+1}{l(l+1)}\left[a_{l} \tau_{l}(\theta)+b_{l} \pi_{l}(\theta)\right] .
\end{aligned}
$$

The angular functions are

$$
\begin{aligned}
\pi_{l}(\theta) & =\frac{1}{\sin \theta} P_{l}^{1}(\cos \theta), \\
\tau_{l}(\theta) & =\frac{\mathrm{d}}{\mathrm{d} \theta} P_{l}^{1}(\cos \theta) .
\end{aligned}
$$

The partial-wave-scattering amplitudes $a_{l}$ and $b_{l}$ are given by the expressions

$$
\left.\begin{array}{l}
a_{l} \\
b_{l}
\end{array}\right\}=\frac{N_{l}^{23}}{N_{l}^{23}+i D_{l}^{23}}
$$


where

$$
\begin{aligned}
N_{l}^{23}= & n_{2} x_{23}^{2}\left[j_{l}\left(x_{23}\right) j_{l-1}\left(n_{2} x_{23}\right)-n_{2} j_{l-1}\left(x_{23}\right) j_{l}\left(n_{2} x_{23}\right)\right. \\
& \left.+\frac{\left(n_{2}^{2}-1\right) l}{n_{2} x_{23}} j_{l}\left(x_{23}\right) j_{l}\left(n_{2} x_{23}\right)\right] \\
& +A_{l} n_{2} x_{23}^{2}\left[j_{l}\left(x_{23}\right) n_{l-1}\left(n_{2} x_{23}\right)\right. \\
& -n_{2} j_{l-1}\left(x_{23}\right) n_{l}\left(n_{2} x_{23}\right) \\
& \left.+\frac{\left(n_{2}^{2}-1\right) l}{n_{2} x_{23}} j_{l}\left(x_{23}\right) n_{l}\left(n_{2} x_{23}\right)\right]
\end{aligned}
$$

$$
D_{l}^{23}=n_{2} x_{23}^{2}\left[n_{l}\left(x_{23}\right) j_{l-1}\left(n_{2} x_{23}\right)-n_{2} n_{l-1}\left(x_{23}\right) j_{l}\left(n_{2} x_{23}\right)\right.
$$$$
\left.+\frac{\left(n_{2}^{2}-1\right) l}{n_{2} x_{23}} n_{l}\left(x_{23}\right) j_{l}\left(n_{2} x_{23}\right)\right]
$$$$
+A_{l} n_{2} x_{23}{ }^{2}\left[n_{l}\left(x_{23}\right) n_{l-1}\left(n_{2} x_{23}\right)-n_{2} n_{l-1}\left(x_{23}\right)\right.
$$$$
\left.\times n_{l}\left(n_{2} x_{23}\right)+\frac{\left(n_{2}^{2}-1\right) l}{n_{2} x_{23}} n_{l}\left(x_{23}\right) n_{l}\left(n_{2} x_{23}\right)\right],
$$

$$
A_{l}=-\frac{N_{l}^{12}}{D_{l}^{12}}
$$

$$
\begin{aligned}
N_{l}^{12}= & n_{1} n_{2} x_{12}{ }^{2}\left[n_{2} j_{l}\left(n_{2} x_{12}\right) j_{l-1}\left(n_{1} x_{12}\right)-n_{1} j_{l-1}\left(n_{2} x_{12}\right)\right. \\
& \left.\times j_{l}\left(n_{1} x_{12}\right)+\frac{\left(n_{1}^{2}-n_{2}^{2}\right) l}{n_{1} n_{2} x_{12}} j_{l}\left(n_{2} x_{12}\right) j_{l}\left(n_{1} x_{12}\right)\right],
\end{aligned}
$$

$$
\begin{aligned}
D_{l}^{12}= & n_{1} n_{2} x_{12}^{2}\left[n_{2} n_{l}\left(n_{2} x_{12}\right) j_{l-1}\left(n_{1} x_{12}\right)-n_{1} n_{l-1}\left(n_{2} x_{12}\right)\right. \\
& \left.\times j_{l}\left(n_{1} x_{12}\right)+\frac{\left(n_{1}^{2}-n_{2}^{2}\right) l}{n_{1} n_{2} x_{12}} n_{l}\left(n_{2} x_{12}\right) j_{l}\left(n_{1} x_{12}\right)\right]
\end{aligned}
$$

for $a_{l}$, and

$$
\begin{aligned}
N_{l}^{23}= & n_{2} x_{23}^{2}\left[n_{2} j_{l}\left(x_{23}\right) j_{l-1}\left(n_{2} x_{23}\right)-j_{l-1}\left(x_{23}\right) j_{l}\left(n_{2} x_{23}\right)\right] \\
& +B_{l} n_{2} x_{23}{ }^{2}\left[n_{2} j_{l}\left(x_{23}\right) n_{l-1}\left(n_{2} x_{23}\right)\right. \\
& \left.-j_{l-1}\left(x_{23}\right) n_{l}\left(n_{2} x_{23}\right)\right] \\
D_{l}^{23}= & n_{2} x_{23}^{2}\left[n_{2} n_{l}\left(x_{23}\right) j_{l-1}\left(n_{2} x_{23}\right)-n_{l-1}\left(x_{23}\right) j_{l}\left(n_{2} x_{23}\right)\right] \\
& +B_{l} n_{2} x_{23}{ }^{2}\left[n_{2} n_{l}\left(x_{23}\right) n_{l-1}\left(n_{2} x_{23}\right)\right. \\
& \left.-n_{l-1}\left(x_{23}\right) n_{l}\left(n_{2} x_{23}\right)\right]
\end{aligned}
$$

$$
\begin{aligned}
B_{l}= & -\frac{N_{l}^{12}}{D_{l}^{12}}, \\
N_{l}^{12}= & n_{1} n_{2} x_{12}^{2}\left[n_{1} j_{l}\left(n_{2} x_{12}\right) j_{l-1}\left(n_{1} x_{12}\right)\right. \\
& \left.-n_{2} j_{l-1}\left(n_{2} x_{12}\right) j_{l}\left(n_{1} x_{12}\right)\right], \\
D_{l}^{12}= & n_{1} n_{2} x_{12}^{2}\left[n_{1} n_{l}\left(n_{2} x_{12}\right) j_{l-1}\left(n_{1} x_{12}\right)\right. \\
& \left.-n_{2} n_{l-1}\left(n_{2} x_{12}\right) j_{l}\left(n_{1} x_{12}\right)\right]
\end{aligned}
$$

for $b_{l}$, and where $j_{l}$ and $n_{l}$ are spherical Bessel functions and spherical Neumann functions, respectively. The principal goal of this paper is to obtain a physical understanding of some of the rainbow phenomena hidden in these complicated expressions.

We first consider the situation in which $x_{12}$ is of the order of a few thousand so that the ray theory is expected to be a good approximation ${ }^{16}$ to Eqs. (4)-(18). We wish for the coating to act as a thin film on the surface of the core. As is proved in Section 3, the thin-film assumption corresponds to $\delta / a_{12} \leq 2 \times$ $10^{-3}$. The two dominant Descartes rays for the first-order rainbow for this situation are shown in Fig. 1(b). The ray that we label $\alpha$ has its internal reflection at the core-coating interface, and the ray that we label $\beta$ has its internal reflection at the coating-air interface. As the core is large, the corecoating-air geometry in the vicinity of the internal reflections is locally flat compared with the wavelength of light. This is illustrated in Fig. 2(a). As a result, the transmissions and the internal reflections may be parameterized by the flat interface electricfield Fresnel coefficients ${ }^{17} T_{g}{ }^{i j}$ and $R_{g}{ }^{i j}$, where first superscript $i$ denotes the initial medium, second superscript $j$ denotes the final medium, and subscript $g$ denotes that this is for a geometric light ray. For reflections within the coating, $R_{g}{ }^{232}$ and $R_{g}{ }^{212}$ denote reflection from medium 2 , off medium 3 or 1 , respectively, and back into medium 2 . As the coating is thin, the $\alpha$ and the $\beta$ rays exit the sphere in the same direction and interfere with each other on the way to the observer.

The Descartes rainbow ray is incident upon the coated sphere at the angle

$$
\cos \theta_{3}^{R}=\left(\frac{n_{1}^{2}-1}{3}\right)^{1 / 2} .
$$

The $\alpha$ and the $\beta$ rainbow rays constructively interfere at the observer, which gives the maximum rainbow intensity if ${ }^{18}$

$$
\frac{4 \pi n_{2} \delta}{\lambda} \cos \theta_{2}^{R}+\phi_{12}+\phi_{23}=2 \pi N,
$$

and they destructively interefere, which gives the minimum rainbow intensity if

$$
\frac{4 \pi n_{2} \delta}{\lambda} \cos \theta_{2}^{R}+\phi_{12}+\phi_{23}=2 \pi(N+1 / 2)
$$




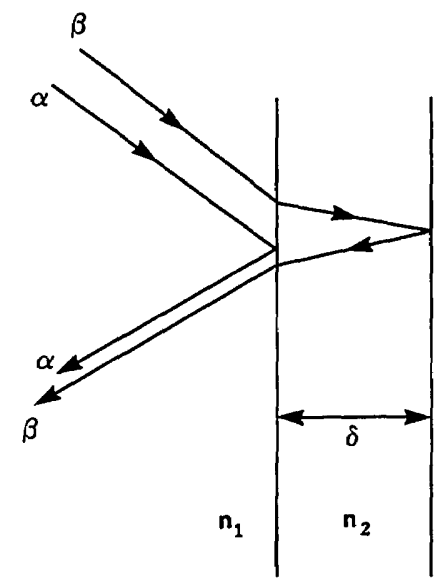

(a)

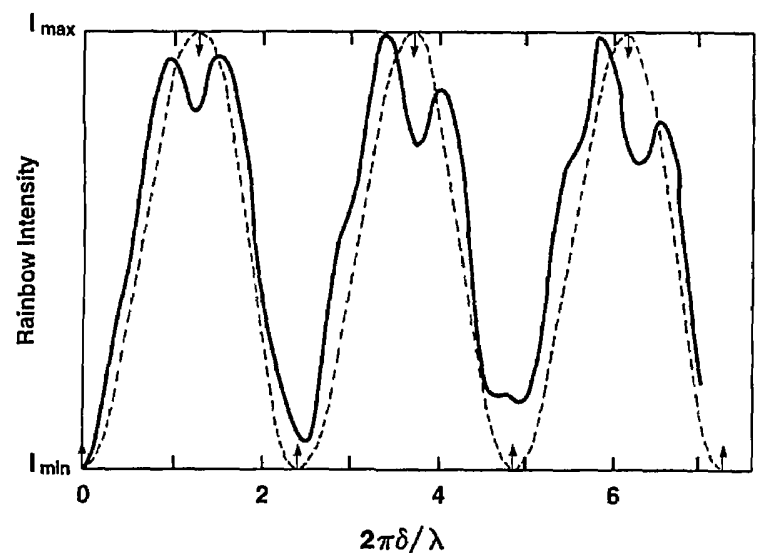

(b)

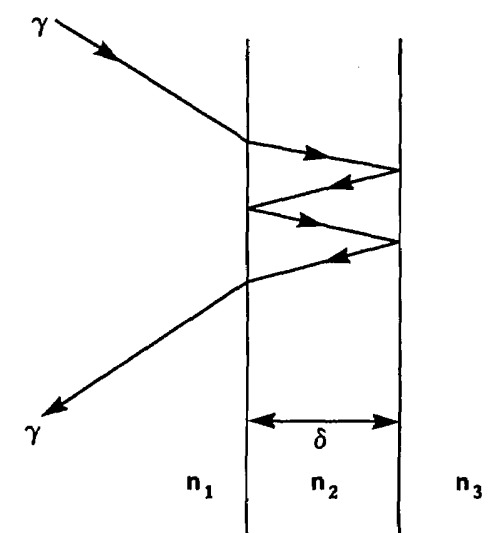

(c)

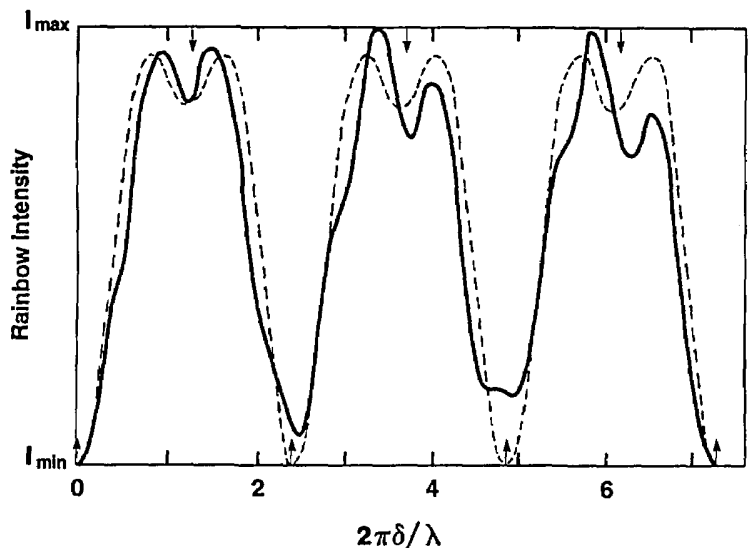

(d)

Fig. 2. (a) When the core is large and the coating is thin, the core-coating-air interfaces are approximated by the thin-film geometry. (b) Comparison between Aden-Kerker wave theory (solid curve) and ray theory employing the $\alpha$ and the $\beta$ rays (dashed curve) for the rainbow intensity as a function of coating thickness. The Aden-Kerker intensity was calculated for $2 \pi \delta / \lambda$ in increments of 0.1 . The arrows denote the coating thicknesses for constructive and destructive interferences in ray theory as predicted by Eqs. (20) and (21), respectively. (c) Ray $\gamma$ that makes three internal reflections within the coating. (d) Comparison between Aden-Kerker wave theory (solid curve) and ray theory when the $\alpha, \beta, \gamma, \gamma^{\prime}$, and $\gamma^{\prime \prime}$ rays (dashed curve) are used for the rainbow intensity as a function of coating thickness.

where $N$ is an integer. The angle $\theta_{2}{ }^{R}$ of the Descartes ray in the coating is given by Snell's law,

$$
n_{3} \sin \theta_{3}{ }^{R}=n_{2} \sin \theta_{2}{ }^{R},
$$

and the phase changes on reflection at the 12 (corecoating) and 23 (coating-air) interfaces are given by

$$
\begin{aligned}
\phi_{12} & = \begin{cases}0 & \text { if } n_{2}<n_{1} \\
\pi & \text { if } n_{2}>n_{1}\end{cases} \\
\phi_{23} & =\left\{\begin{array}{ll}
0 & \text { if } n_{3}<n_{2} \\
\pi & \text { if } n_{3}>n_{2}
\end{array} .\right.
\end{aligned}
$$

As a specific example, we consider $n_{1}=1.333, n_{2}=$ 1.55 , and $n_{3}=1.0$, which describes a thin-wall glass shell filled with water. The amplitude of the $\alpha$ rainbow ray is given by

$$
E_{\alpha}=T_{g}{ }^{32} T_{g}{ }^{21} R_{g}{ }^{11} T_{g}{ }^{12} T_{g}{ }^{23}=0.0941
$$

for the transverse electric (TE) polarization, and the amplitude of the $\beta$ rainbow ray is given by

$$
E_{\beta}=T_{g}{ }^{32} T_{g}{ }^{21} T_{g}{ }^{12} R_{g}{ }^{232} T_{g}{ }^{21} T_{g}{ }^{12} T_{g}{ }^{23}=0.3425
$$

for the TE polarization. Assuming that the only difference in the relative strengths of $\alpha$ and $\beta$ rainbows is given by the different sets of Fresnel coefficients in Eqs. (24) and (25), the maximum rainbow intensity is

$$
I_{\max }=\frac{1}{2 \mu_{0} c}\left(E_{\alpha}+E_{\beta}\right)^{2}=0.1906,
$$

which corresponds to constructive interference of the $\alpha$ and the $\beta$ rays, and the minimum rainbow intensity is

$$
I_{\min }=\frac{1}{2 \mu_{0} c}\left(E_{\alpha}-E_{\beta}\right)^{2}=0.0617,
$$

which corresponds to destructive interference. This produces an intensity contrast factor of $I_{\max } / I_{\min }=$ 3.1. 
To verify this ray-theory oscillating intensity prediction, we computed the wave-theory intensity of Eqs. (4)-(18) for $x_{12}=3000$ and $0 \leq(2 \pi \delta) / \lambda \leq 7$. In each case the scattered intensity between $135^{\circ} \leq \theta \leq$ $140^{\circ}$ was Gaussian low-pass filtered in order to subtract off the high-spatial-frequency fine structure superposed on the rainbow intensity caused by the interference of specularly reflected rays with the rays that make one internal reflection. The peak rainbow intensity of the filtered data was then determined and is shown in Fig. 2(b). The wave-theory intensity contrast factor is found to be $I_{\max } / I_{\min }=1.6$, which is in disagreement with the ray-theory result just obtained. The background intensity upon which the rainbow is superposed was only $\sim 1 \%$ of the total intensity at the rainbow peak, thus not influencing the value of $I_{\max } / I_{\min }$. As a check on our method of calculation, the intensity in Alexander's dark band, which is due primarily to specular reflection, was also calculated both in ray theory and in Aden-Kerker wave theory. The dominant geometric rays for specular reflection are parameterized by $R_{g}{ }^{33}$ and by $T_{g}{ }^{32} R_{g}{ }^{212} T_{g}{ }^{23}$. These rays also alternately constructively and destructively interefere as $\delta$ increases. The specular reflection intensity contrast factor in Alexander's dark band was found to have the values of 3.9 in ray theory and 4.0 in Aden-Kerker theory. The agreement for specular reflection and disagreement for the first-order rainbow lead us to believe that the extent of the partial focusing of light rays that occurs at the first-order rainbow and causes its enhancement above the geometric optics background is affected by the presence of the coating.

In Fig. 2(b), we also show the ray-theory intensity that is due to the $\alpha$ and the $\beta$ rays normalized to the Aden-Kerker results. The normalized comparison is generally good except for the double-peak structure at the constructive interference coating thicknesses in wave theory. This discrepancy is removed when we take into account in ray theory the geometric rays that make three internal reflections within the coating. There are three distinct ray paths that do this. One of them, which we call the $\gamma$ ray, is shown in Fig. 2(c). The other two rays $\left(\gamma^{\prime}\right.$ and $\left.\gamma^{\prime \prime}\right)$ resemble the $\beta$ ray but have two additional internal reflections in the coating immediately after entering the coating for the first time or immediately before exiting it for the last time.

The normalized comparison between wave theory and ray theory when the $\alpha, \beta, \gamma, \gamma^{\prime}$, and $\gamma^{\prime \prime}$ rays are used is shown in Fig. 2(d). The inclusion of the $\gamma, \gamma^{\prime}$, and $\gamma^{\prime \prime}$ rays reproduces the wave-theory double-peak structure at the constructive interference locations. But the double peak in ray theory is not as prominent as that of the Aden-Kerker wave theory. If the $\gamma, \gamma^{\prime}$, and $\gamma^{\prime \prime}$ amplitudes are increased by $18 \%$ over the values given by the Fresnel coefficients, which is the situation graphed in Fig. 2(d), the agreement is much improved. This may be taken as additional evidence that the coating perturbs the degree of partial focusing that occurs for the coated-sphere rainbow beyond that described by the Fresnel coefficients. As a final check on the $\gamma, \gamma^{\prime}$, and $\gamma^{\prime \prime}$ rays' being responsible for the double-peak structure in Figs. 2(b) and 2(d), calculations were also performed for $n_{1}=1.333, n_{2}=$ 1.2 , and $n_{3}=1.0$. When $n_{2}<n_{1}$, both in AdenKerker theory and in ray theory with the $\alpha, \beta, \gamma, \gamma^{\prime}$, and $\gamma^{\prime \prime}$ rays, there is only a single peak at the constructive interference locations for realistic amplitudes of the $\gamma, \gamma^{\prime}$, and $\gamma^{\prime \prime}$ rays, which indicates the appropriateness of including these contributions in Fig. 2(d).

\section{Twin First-Order Rainbows}

In Fig. 1 (a) the angle $\theta_{2}{ }^{\prime}$ is given by

$$
\begin{aligned}
\theta_{2}{ }^{\prime}= & \theta_{2} \\
& +\arccos \left[\frac{a_{23}}{a_{12}} \sin ^{2} \theta_{2}+\cos \theta_{2}\left(1-\frac{a_{23}{ }^{2}}{a_{12}{ }^{2}} \sin ^{2} \theta_{2}\right)^{1 / 2}\right] \\
\approx & \theta_{2}+\frac{\delta}{a_{12}} \sin \theta_{3}\left(n_{2}^{2}-\sin ^{2} \theta_{3}\right)^{-1 / 2}+\mathscr{O}\left(\frac{\delta^{2}}{a_{12}{ }^{2}}\right) \cdot
\end{aligned}
$$

As the coating grows in thickness, the difference between the angles $\theta_{2}$ and $\theta_{2}{ }^{\prime}$ increases as well. Because the $\alpha$ ray makes two transits through the coating whereas the $\beta$ ray makes four transits, the two rays leave the sphere at different scattering angles and thus produce two distinct first-order rainbows. Let

$$
\epsilon=\theta_{2}{ }^{\prime}-\theta_{2} \text {. }
$$

Then the angular deviation of the $\alpha$ ray is

$$
\theta_{\alpha}=\pi+2 \theta_{3}-4 \theta_{2}+2 \epsilon,
$$

and the angular deviation of the $\beta$ ray is

$$
\theta_{\beta}=\pi+2 \theta_{3}-4 \theta_{2}+4 \epsilon .
$$

The derivatives of $\theta_{\alpha}$ and $\theta_{\beta}$ were performed with respect to $\theta_{3}$ and then set equal to zero in order to determine the angle $\theta_{3}$ of the incident rainbow ray. The resulting expressions, however, cannot be solved in closed form. To obtain an approximate solution, we Taylor-series expanded all the angles in powers of $\delta / a_{12}$ about their values for the ray path of the homogeneous-sphere Descartes ray. The results were then substituted into Eqs. (30) and (31) to obtain the rainbow scattering angle of the $\alpha$ and the $\beta$ rays:

$$
\begin{aligned}
\Theta_{\alpha}{ }^{R}\left(\frac{\delta}{a_{12}}\right)= & \Theta^{R}(0)+\frac{2 \delta}{a_{12}}\left[\left(\frac{4-n_{1}^{2}}{3 n_{2}^{2}+n_{1}^{2}-4}\right)^{1 / 2}\right. \\
& \left.-\left(\frac{4-n_{1}^{2}}{n_{1}^{2}-1}\right)^{1 / 2}\right]+\mathscr{O}\left(\frac{\delta^{2}}{a_{12}^{2}}\right), \\
\Theta_{\beta}{ }^{R}\left(\frac{\delta}{a_{12}}\right)= & \Theta^{R}(0)+\frac{2 \delta}{a_{12}}\left[2\left(\frac{4-n_{1}^{2}}{3 n_{2}^{2}+n_{1}^{2}-4}\right)^{1 / 2}\right. \\
& \left.-\left(\frac{4-n_{1}^{2}}{n_{1}^{2}-1}\right)^{1 / 2}\right]+\mathscr{O}\left(\frac{\delta^{2}}{a_{12}^{2}}\right),
\end{aligned}
$$


where $\theta^{R}(0)$ is the rainbow-scattering angle for the homogeneous sphere. For the specific example $n_{1}=$ 1.333, $n_{2}=1.2, n_{3}=1.0$, and $x_{12}=5000$, this twinning and shifting of the rainbow-scattering angle is shown in Fig. 3(a) as a function of $(2 \pi \delta) / \lambda$. The thin-film limit examined in Section 2 for $(2 \pi \delta) / \lambda \leq 7$ corresponds to the far left-hand side of the graph, where the $\alpha$ and the $\beta$ rainbows are shifted by only minute amounts from the homogeneous-sphere rainbow. Thus it was reasonable in Section 2 to consider the exiting rays as coincident and interfering with each other. For larger coating thicknesses, the two rainbow rays no longer spatially overlap and thus can no longer interfere.

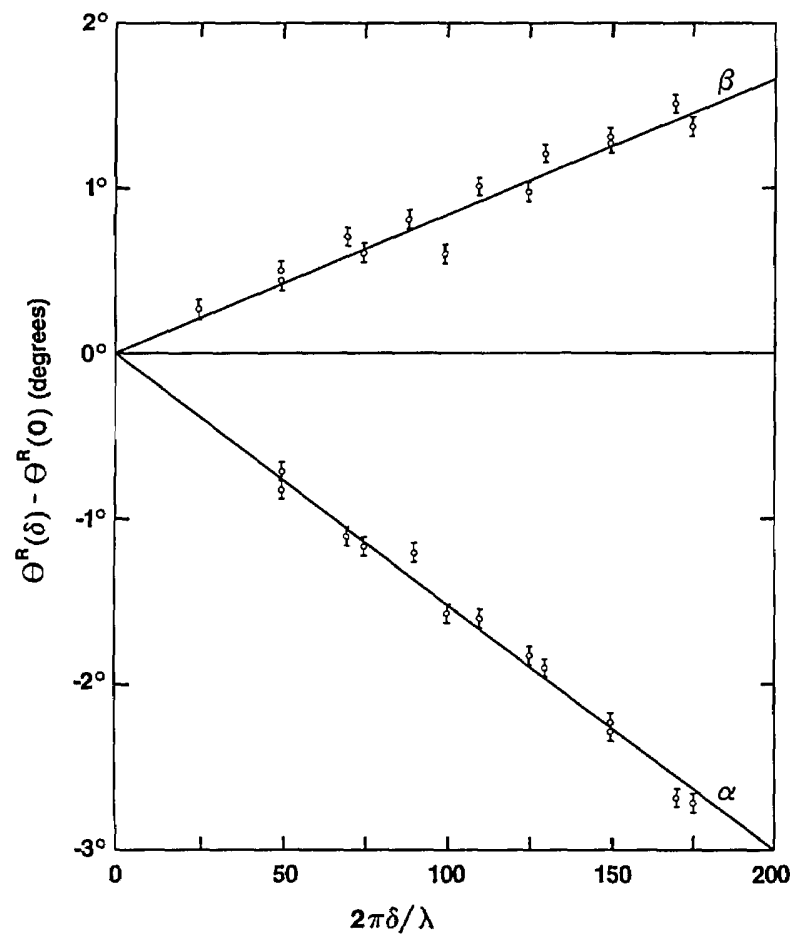

(a)

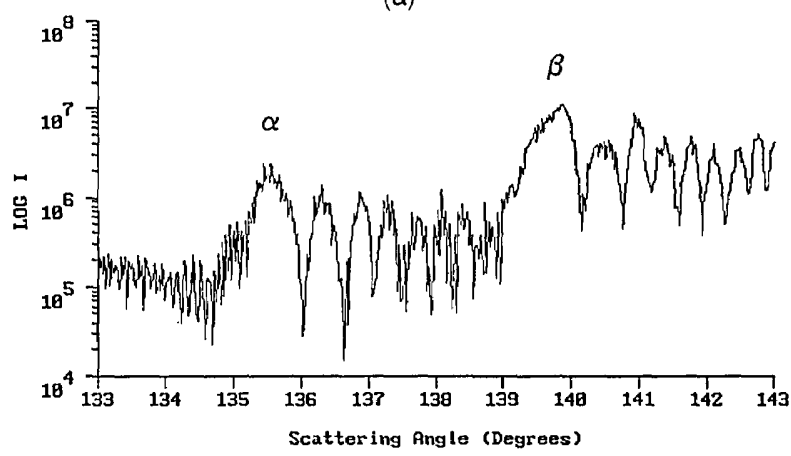

(b)

Fig. 3. (a) Comparison between Aden-Kerker wave theory (data points) and ray theory (solid lines) for the shift in the angular positions of the twin first-order rainbows as a function of coating thickness for the $\alpha$ and the $\beta$ rainbows of Fig. 1(b). (b) Scattered intensity for unpolarized incident light as a function of $\Theta$ for $x_{12}=$ $5000,(2 \pi \delta) / \lambda=175, n_{1}=1.333, n_{2}=1.2$, and $n_{3}=1.0$, showing the twin first-order rainbows $\alpha$ and $\beta$ of Fig. 1(b).
Again, to test the ray theory result, we calculated the wave-theory intensity from Eqs. (4)-(18). A typical example is shown in Fig. $3(\mathrm{~b})$. The $\alpha$ rainbow occurs at $\theta \approx 135.5^{\circ}$, and the $\beta$ rainbow occurs at $\theta \approx 139.5^{\circ}$. For $25 \leq(2 \pi \delta) / \lambda \leq 175$, the computed angular positions of the $\alpha$ and the $\beta$ rainbows obtained from the Aden-Kerker equations were determined and are shown as the data points in Fig. 3(a). The error bars correspond to the uncertainty in determining the maximum rainbow intensity. The comparison between ray theory and wave theory is quite good. However, the rainbow-intensity contrast factors for ray theory and wave theory again disagree, with $I_{\beta} / I_{\alpha}=13.24$ in ray theory and $I_{\beta} / I_{\alpha}=$ 4.8 in wave theory. This is again presumably due to the effect the coating has on the degree of partial focusing at the rainbow. The $\gamma, \gamma^{\prime}$, and $\gamma^{\prime \prime}$ rainbows are not evident in Fig. 3(b). This is because their amplitude is much less than that of the $\alpha$ and the $\beta$ rainbows and because they are located in the supernumeraries of the $\beta$ rainbow.

Calculations were also performed for $n_{1}=1.333$, $n_{2}=1.55$, and $n_{3}=1.0$. Good agreement for the rainbow-scattering angles between Aden-Kerker theory and ray theory was again obtained. Estimates of the angles, however, were more difficult to obtain because of the larger amplitude interference structure superposed on the $\alpha$ rainbow for $n_{2}>n_{1}$.

\section{Debye-Series Analysis of the Coated-Sphere Scattering Amplitudes}

In Mie theory the incident wave, scattered wave, and interior wave are decomposed into partial waves. The resulting infinite series solution to the scattering problem for a homogeneous sphere is exact. But it gives little clue as to the various physical processes responsible for the scattering. These physical processes are diffraction, specular reflection $(p=0)$, transmission $(p=1)$, and transmission following $p-$ 1 internal reflections. They appear naturally in ray theory but manifest themselves in Mie theory only when various numbers of interactions of each partial wave with the particle surface are considered. As it turns out, each partial-wave scattering amplitude may be written as a sum of components that correspond to diffraction of the partial wave, specular reflection of the partial wave, transmission of the partial wave, and transmission of the partial wave following $p-1$ internal reflections as

$$
\begin{aligned}
& \left.\begin{array}{l}
a_{l} \\
b_{l}
\end{array}\right\}=\frac{1}{2}\left(1-R_{l}^{22}-\frac{T_{l}^{21} T_{l}^{12}}{1-R_{l}^{11}}\right) \\
& =\frac{1}{2}\left(1-R_{l}^{22}-T_{l}^{21} T_{l}^{12}-T_{l}^{21} R_{l}^{11} T_{l}^{12}-\cdots\right) .
\end{aligned}
$$

This procedure is known as the Debye-series decomposition of the partial-wave scattering amplitudes. Expressions for $R_{l}{ }^{i j}$ and $T_{l}{ }^{i j}$, the reflection and the 
transmission amplitudes of the partial wave $l$, are given elsewhere..$^{8-11}$ Written in this form, the solution of the homogeneous-sphere scattering problem is obtained by summing over both partial waves $l$ and scattering processes $p$. The general success of the ray-theory and the wave-theory comparisons of Sections 2 and 3 gives hope that a Debye-series analysis of the coated-sphere problem is not only possible, but that it provides valuable physical insight into the details of coated-sphere scattering. We derive the coated-sphere Debye series in this section.

As a guide for what to expect, we first consider multiple scattering of geometric light rays within a coated sphere. The first number of terms in the multiple-scattering series is pictorially illustrated in Fig. 4. For a given number of total interactions with the core-coating and the coating-air interfaces, there are many possible ray paths. Some ray paths involve different sets of Fresnel coefficients (for example, $T_{g}{ }^{32} T_{g}{ }^{21} T_{g}{ }^{12} T_{g}{ }^{23}$ and $T_{g}{ }^{32} R_{g}{ }^{232} R_{g}{ }^{232} T_{g}{ }^{23}$ ), and others involve the same Fresnel coefficients but are taken in different orders (for example, $T_{g}{ }^{32} R_{g}{ }^{212} R_{g}{ }^{232} T_{g}{ }^{23}$ and $\left.T_{g}{ }^{32} R_{g}{ }^{232} R_{g}{ }^{212} T_{g}{ }^{23}\right)$. When all the possible ray paths are included, the multiple-scattering series is analytically sumable, and the ray-theory scattered electric field is given by

$$
E_{g}=D+R_{g}^{33}+\frac{T_{g}{ }^{32}\left(Q_{g}+1\right) T_{g}{ }^{23}}{1-R_{g}{ }^{232}\left(Q_{g}+1\right)}
$$

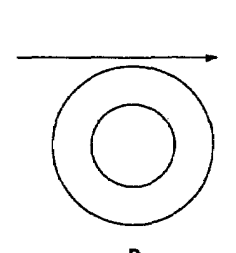

D
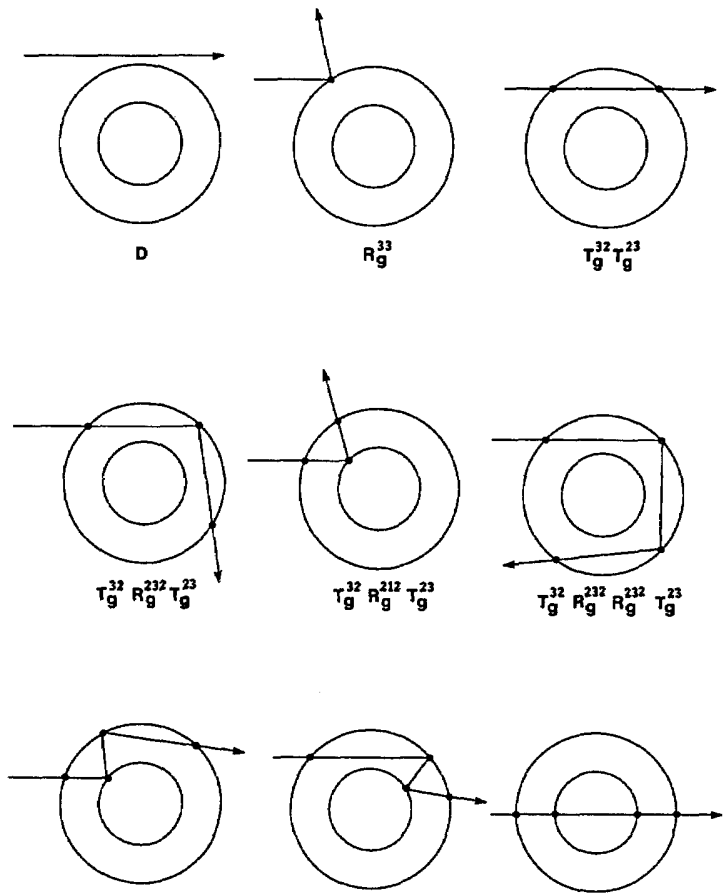

$T_{g}^{32} R_{g}^{212} R_{g}^{232} T_{g}^{23}$
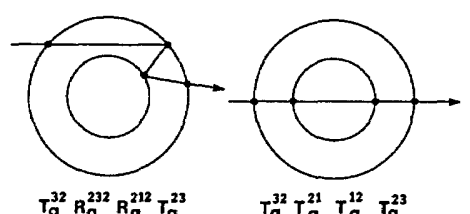

$T_{g}^{32} T_{g}^{21} T_{g}^{12} T_{g}^{23}$
Fig. 4. Ray trajectories with up to four interactions with the interfaces that contribute to geometric ray scattering by a coated sphere. where

$$
Q_{g}=R_{g}{ }^{212}+\frac{T_{g}{ }^{11} T_{g}{ }^{12}}{1-R_{g}{ }^{11}}
$$

and $D$ symbolically represents diffraction by the composite sphere. The multiple-scattering series is recovered by expanding the denominators of Eqs. (35) and (36). Equation (36) represents the infinite series of interactions of the ray with the core alone. The $T_{g}{ }^{32}, T_{g}{ }^{23}$, and $R_{g}{ }^{232}$ factors in the last term of Eq. (35) describe the interaction of the rays with the coating before they penetrate into the core and after they exit it. Geometric light rays are allowed to interact with the coating-air interface a number of times successively. This is described by the factor of 1 in the $Q_{g}+1$ expressions. If diffraction by the core (which is represented by $d$ ) were to occur between such successive coating-air interactions, the factors of $Q_{g}+1$ in Eq. (35) would be replaced by $Q_{g}+d$.

Although each light ray travels in a straight line between interactions with the interfaces, the radial dependence of a partial wave is $h_{l}^{(1)}(k r)$ or $h_{l}^{(2)}(k r)$, where $h_{l}$ are spherical Hankel functions, which implies that partial waves propagate radially outward or inward. ${ }^{19}$ This radial motion forbids successive interactions of partial waves with the coating-air interface. Not surprisingly then and after much algebra, the Debye-series decomposition of the coated-sphere partial-wave scattering amplitudes is found to be $\mathrm{e}^{20,21}$

$$
\left.\begin{array}{c}
a_{l} \\
b_{l}
\end{array}\right\}=\frac{1}{2}\left(1-R_{l}^{33}-\frac{T_{l}^{32} Q_{l} T_{l}^{23}}{1-Q_{l} R_{l}^{232}}\right),
$$

where

$$
Q_{l}=R_{l}^{212}+\frac{T_{l}^{21} T_{l}^{12}}{1-R_{l}^{11}}
$$

The meaning of the transmission and the reflection amplitudes $R_{l}^{33}, T_{l}^{32}, T_{l}^{23}, R_{l}^{232}, R_{l}{ }^{212}, T_{l}^{21}, T_{l}{ }^{12}$, and $R_{l}{ }^{11}$ for the partial wave $l$ is the same as in the homogeneous-sphere case of Eq. (34). Equation (38) is, with the exception of diffraction, the scattering amplitude of the core in an infinite extent of coating material. Similarly, with the exception of the $Q_{l}$ factors, Eq. (37) is the scattering amplitude of a particle that comprises coating material in an infinite extent of medium 3. The $Q_{l}$ factors in Eq. (37) give the influence of the core on composite sphere scattering. The first few terms in Eqs. (37) and (38) are pictorially illustrated in Figs. 5. The star symbol within the core defined in Fig. 5(a) represents the infinite series of successive interactions of the partial wave with the coating-core interface before it heads back into the coating.

In the derivation of the Aden-Kerker equations, the scattered electric and magnetic fields are obtained 


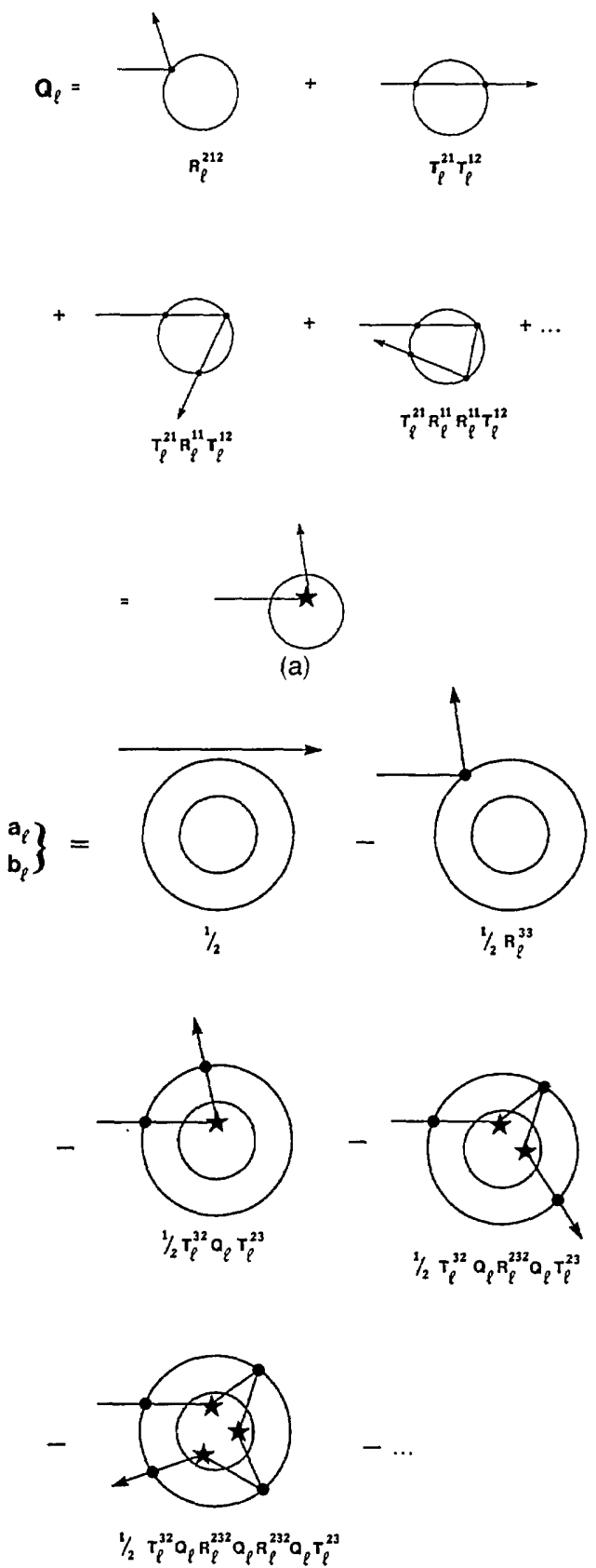

(b)

Fig. 5. (a) Debye-series expansion of the core scattering amplitude $Q_{l}$ of Eq. (38). (b) Debye-series expansion of the coated-sphere partial-wave-scattering amplitudes $a_{l}$ and $b_{l}$ of Eq. (37). The star symbol represents the infinite series of successive interactions of the partial wave with the coating-core interface beafore it heads back into the coating.

by differentiation of the scalar radiation potentials:

$$
\begin{aligned}
\psi_{\text {scattered }}^{\mathrm{TE}} & =\sum_{l=1}^{\infty} i^{l} \frac{2 l+1}{l(l+1)}\left(-b_{l}\right) h_{l}^{(1)}(k r) P_{l}(\cos \theta) \sin \phi, \\
\psi_{\text {scattered }}^{\mathrm{TM}} & =\sum_{l=1}^{\infty} i^{l} \frac{2 l+1}{l(l+1)}\left(-a_{l}\right) h_{l}^{(1)}(k r) P_{l}{ }^{1}(\cos \theta) \cos \phi .
\end{aligned}
$$

Similarly, the fields in the core are derived from the scalar radiation potentials:

$$
\begin{aligned}
& \psi_{\mathrm{core}}^{\mathrm{TE}}=\sum_{l=1}^{\infty} i^{l} \frac{2 l+1}{l(l+1)}\left(n_{1} d_{l}\right) j_{l}\left(n_{1} k r\right) P_{l}^{1}(\cos \theta) \sin \phi, \\
& \psi_{\mathrm{core}}^{\mathrm{TM}}=\sum_{l=1}^{\infty} i^{l} \frac{2 l+1}{l(l+1)}\left(n_{1} c_{l}\right) j_{l}\left(n_{1} k r\right) P_{l}^{1}(\cos \theta) \sin \phi,
\end{aligned}
$$

where $c_{l}$ and $d_{l}$ are the partial-wave core amplitudes, and the fields in the coating are derived from the potentials

$$
\begin{aligned}
\psi_{\text {coating }}^{\mathrm{TE}}= & \sum_{l=1}^{\infty} i^{l} \frac{2 l+1}{l(l+1)} n_{2}\left[L_{l}{ }^{\text {out }} h_{l}^{(1)}\left(n_{2} k r\right)+L_{l}^{\text {in }} h_{l}^{(2)}\left(n_{2} k r\right)\right] \\
& \times P_{l}^{1}(\cos \theta) \sin \phi, \\
\psi_{\text {coating }}^{\mathrm{TM}}= & \sum_{l=1}^{\infty} i^{i^{l}} \frac{2 l+1}{l(l+1)} n_{2}\left[K_{l}^{\text {out }} h_{l}^{(1)}\left(n_{2} k r\right)+K_{l}^{\text {in }} h_{l}^{(2)}\left(n_{2} k r\right)\right] \\
& \times P_{l}{ }^{1}(\cos \theta) \cos \phi,
\end{aligned}
$$

where $K_{l}^{\text {out }}, K_{l}^{\text {in }}, L_{l}^{\text {out }}$, and $L_{l}^{\text {in }}$ are the partial-wave coating amplitudes. Expressions for the coating and the core partial-wave amplitudes are given in Ref. 22. When an algebraic calculation similar to the derivation of Eqs. (37) and (38) is employed, the Debyeseries decomposition of the core and the coating partial-wave amplitudes are found to be

$$
\begin{aligned}
& \left.\begin{array}{c}
c_{l} \\
d_{l}
\end{array}\right\}=\frac{n_{3}}{n_{1}} \frac{T_{l}^{32} I_{l}}{1-Q_{l} R_{l}^{232}}, \\
& I_{l}=\frac{T_{l}^{21}}{1-R_{l}^{11}}, \\
& \left.\begin{array}{c}
K_{l}^{\text {in }} \\
L_{l}^{\text {in }}
\end{array}\right\}=\frac{n_{3}}{2 n_{2}} \frac{T_{l}^{32}}{1-Q_{l} R_{l}^{232}}, \\
& \left.\begin{array}{c}
K_{l}^{\text {out }} \\
L_{l}^{\text {out }}
\end{array}\right\}=\frac{n_{3}}{2 n_{2}} \frac{T_{l}^{32} Q_{l}}{1-Q_{l} R_{l}^{232}} .
\end{aligned}
$$

For scattering by a homogeneous sphere, the $I_{l}$ term of Eq. (43) describes a partial wave that has entered the core but has not yet exited it. ${ }^{10}$ It may have entered the core either immediately after being transmitted into the coating $\left[T_{l}^{32}\right.$ in Eq. (42)] or after having bounced back and forth within the coating a number of times $\left[1 /\left(1-Q_{l} R_{l}^{232}\right)\right]$. The amplitudes $K_{l}^{\text {in }}$ and $L_{l}{ }^{\text {in }}$ are associated with the radially incoming Hankel function $h_{l}{ }^{(2)}\left(n_{2} k r\right)$ and represent partial waves that have entered the coating $\left[T_{l}^{32}\right.$ in Eq. (44)] and are heading radially inward toward the core. The amplitudes $K_{l}^{\text {out }}$ and $L_{l}{ }^{\text {out }}$ are associated with the outgoing Hankel function $h_{l}{ }^{(1)}\left(n_{2} k r\right)$ and represent partial waves within the coating that have entered it $\left[T_{l}^{32}\right.$ in Eq. $(45)]$, interacted with the core $\left(Q_{l}\right)$, and are now 


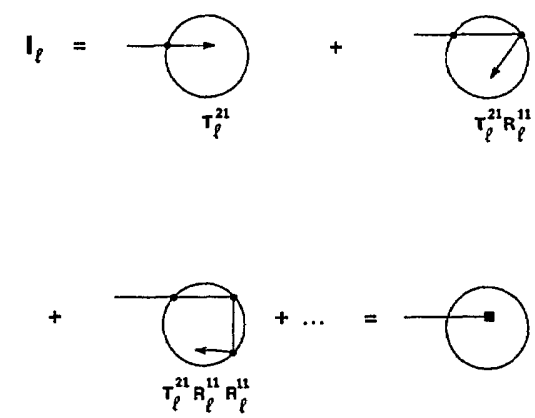

(a)
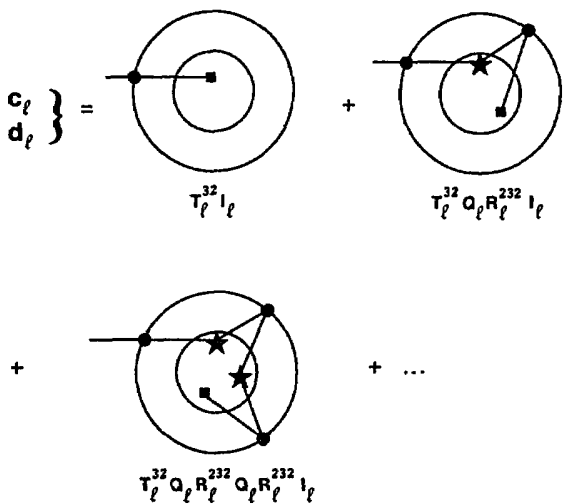

(b)

Fig. 6. (a) Debye-series expansion of the partial-wave interior function $I_{l}$ of Eq. (43). (b) Debye-series expansion of the core partial-wave amplitudes $c_{l}$ and $d_{l}$ of Eq. (42). The filled square represents the infinite series of successive interactions of the partial wave with the coating-core interface before it finally ends up in the core.

heading radially outward toward the coating-air interface. Because the partial waves within the core have the standing-wave radial dependence $j_{l}\left(n_{1} k r\right)$, they represent a superposition of radially incoming and outgoing waves. The first few terms in Eqs. (42)(45) are pictorially illustrated in Figs. 6 and 7. The filled square within the core defined in Fig. 6(a) represents the infinite series of successive interac-

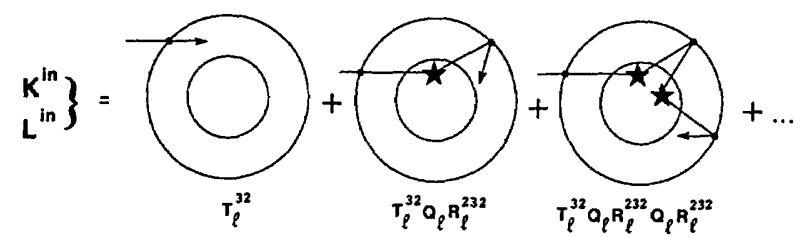

(a)

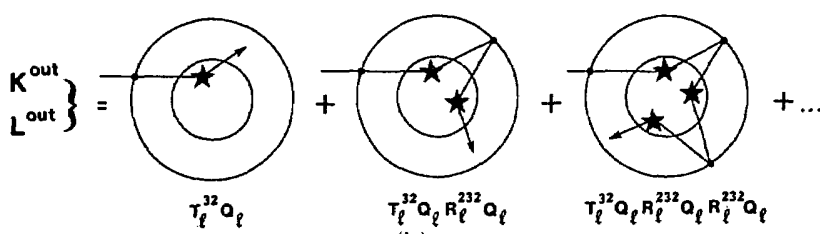

(b)

Fig. 7. (a) Debye-series expansion of the partial-wave coating incoming amplitudes $K_{l}^{\text {in }}$ and $L_{l}^{\text {in }}$ of Eq. (44). (b) Debye-series expansion of the partial-wave coating outgoing amplitudes $K_{l}^{\text {out }}$ and $L_{l}{ }^{\text {out }}$ of Eq. (45).
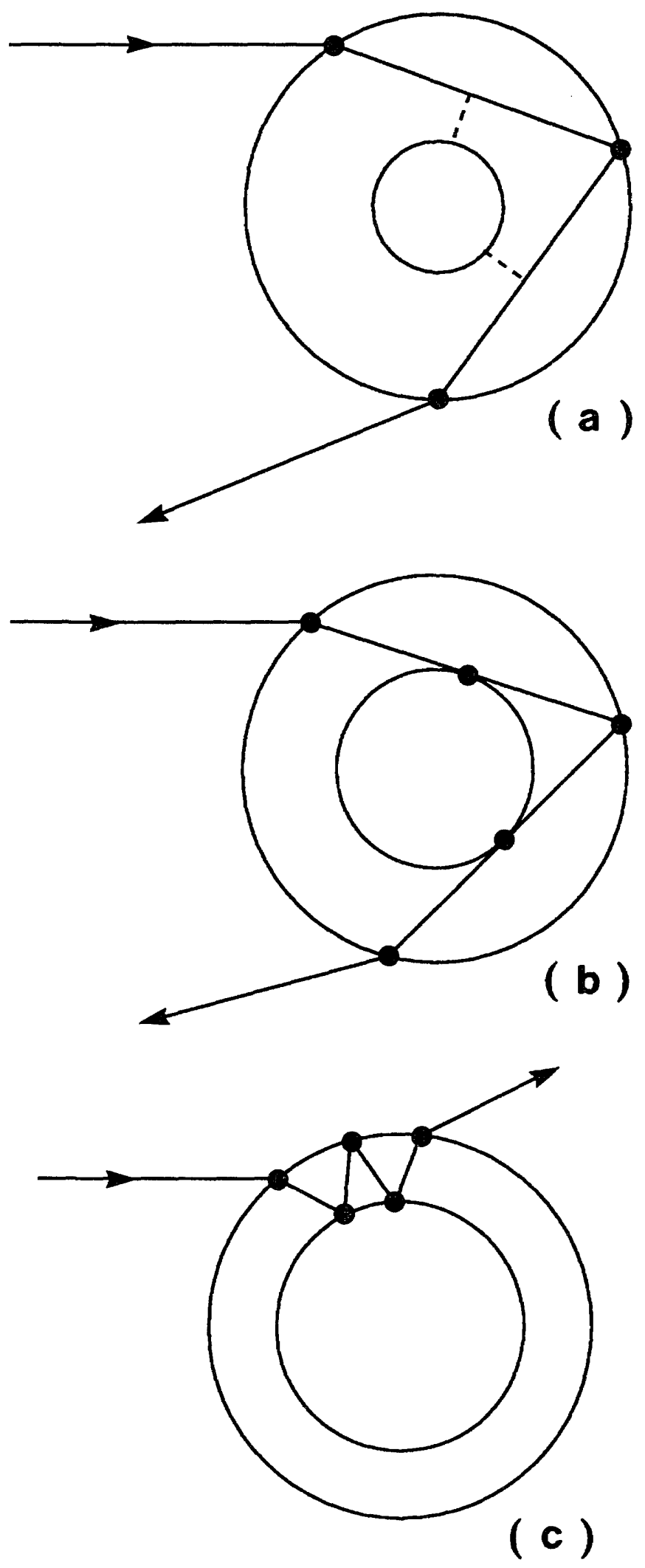

Fig. 8. Ray trajectories corresponding to the Debye-series term $T_{l}^{32} R_{l}^{212} R_{l}{ }^{232} R_{l}{ }^{212} T_{l}{ }^{23}$ for (a) a small core that permits rainbow formation, (b) a core that begins to obstruct the Descartes rainbow ray, causing a rainbow extinction transition, (c) a large core for which no rainbow can occur. The dashed lines in (a) indicate the partial-wave reflection coefficient $R_{l}^{212} \rightarrow 1$. 
tions of the partial wave with the coating-core interface before it finally ends up in the core.

As a final check of these results, the generalized Lorenz-Mie theory describes scattering of an arbitrary incident wave by a spherical particle. ${ }^{12,13}$ If the scalar radiation potential in the coating were written as the sum of a distorted incident standing wave plus an outgoing scattered wave, the coating and the core fields should represent the incident, scattered, and internal fields for scattering of the distorted standing wave in the coating by the spherical core. To test this idea, we rewrite Eq. (41) as

$$
\begin{aligned}
& \psi_{\text {coating }}^{\mathrm{TE}}= \sum_{l=1}^{\infty} i^{l} \frac{2 l+1}{l(l+1)} n_{2}\left[V_{l}^{\text {inc }} j_{l}\left(n_{2} k r\right)-V_{l}^{\text {scatt }} h_{l}^{(1)}\left(n_{2} k r\right)\right] \\
& \times P_{l}^{1}(\cos \theta) \sin \phi, \\
& \psi_{\text {coating }}^{\mathrm{TM}} \sum_{l=1}^{\infty} i^{l} \frac{2 l+1}{l(l+1)} n_{2}\left[U_{l}^{\text {inc }} j_{l}\left(n_{2} k r\right)-U_{l}^{\text {scatt }} h_{l}^{(1)}\left(n_{2} k r\right)\right] \\
& \times P_{l}^{1}(\cos \theta) \cos \phi, \\
& U_{l}^{\text {inc }} \\
&\left.V_{l}^{\text {inc }}\right\}= \frac{n_{3}}{n_{2}} \frac{T_{l}^{32}}{1-Q_{l} R_{l}^{232}}, \\
& U_{l}^{\text {scatt }}= 1 / 2 U_{l}^{\text {inc }}\left(1-Q_{l}\right), \\
& V_{l}^{\text {scatt }}= 1 / 2 V_{l}^{\text {inc }}\left(1-Q_{l}\right),
\end{aligned}
$$

where $V_{l}^{\text {inc }} j_{l}$ and $U_{l}^{\text {inc }} j_{l}$ represent the distorted incident standing wave in the coating, and $-V_{l}{ }^{\text {scatt }} h_{l}{ }^{(1)}$ and $-U_{l}^{\text {scat }} h_{l}^{(1)}$ represent the outgoing scattered wave in the coating. Equations $(46)-(48)$, along with the

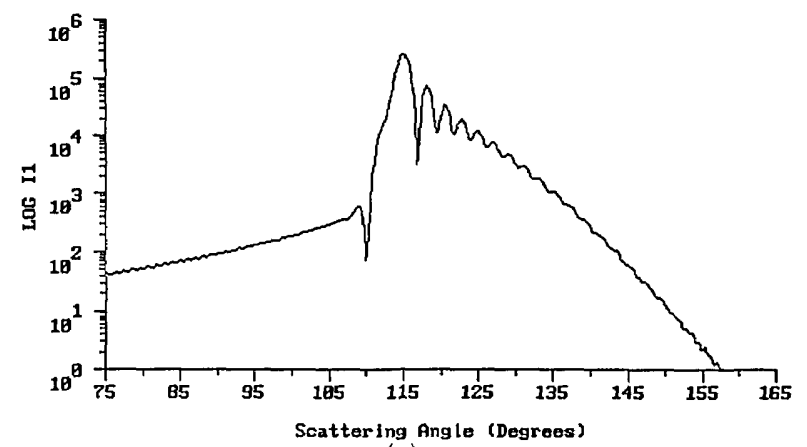

(a)

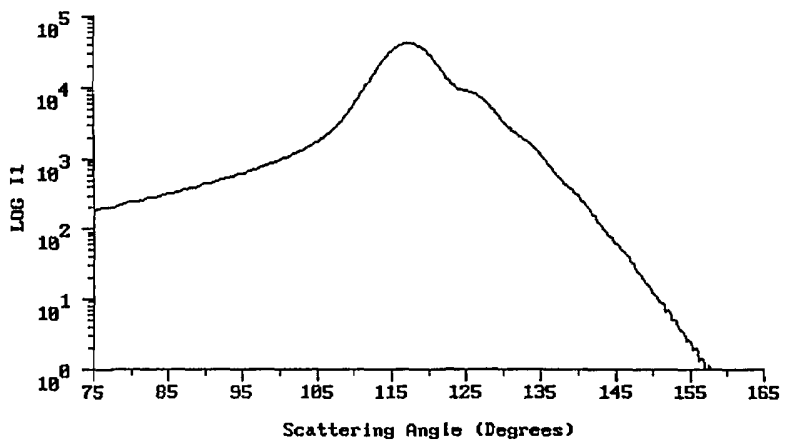

(b) core partial-wave amplitudes rewritten as

$$
\begin{aligned}
c_{l} & =\frac{n_{2}}{n_{1}} U_{l}^{\mathrm{inc}} I_{l}, \\
d_{l} & =\frac{n_{2}}{n_{1}} V_{l}^{\mathrm{inc}} I_{l},
\end{aligned}
$$

are of the form required by the generalized LorenzMie theory.

\section{Rainbow-Extinction Transitions}

When the far-field scattered intensity is calculated with the Aden-Kerker equations, subtle physical effects that are due to multiple internal reflections are often masked by much stronger backgroundscattering mechanisms such as specular reflection and transmission. The Debye series is invaluable in subtracting off the unwanted large background in order to examine in detail the subtle physical effect under consideration. We adopt this subtraction method in this section when studying rainbowextinction transitions. Consider, for example, the ray trajectories of Figs. 8. When the core is small, the Descartes rainbow ray propagates through the coating and misses the core, which permits rainbow formation [Fig. 8(a)]. As the core grows while the composite radius $a_{23}$ stays constant, the core eventually blocks the Descartes ray, extinguishing the rainbow [Fig. 8(b)]. When the core is large and the coating is thin, the coating acts as a waveguide for the incident rays, and rainbow formation is impossible [Fig. 8(c)].

In the Debye series, the wave-theory version of the

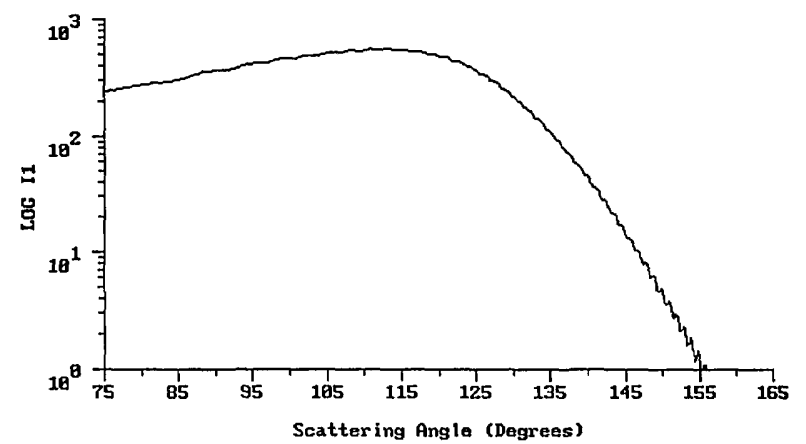

(c)

Fig. 9. Scattered intensity $\left|S_{1}(\theta)\right|^{2}$ of Eq. (50) as a function of $\theta$ for $x_{23}=900, n_{1}=1.333, n_{2}=1.2, n_{3}=1.0$, and (a) $x_{12}=600$ where rainbow formation occurs, (b) $x_{12}=700$ near the rainbow extinction transition, (c) $x_{12}=750$ where rainbow formation does not occur. 


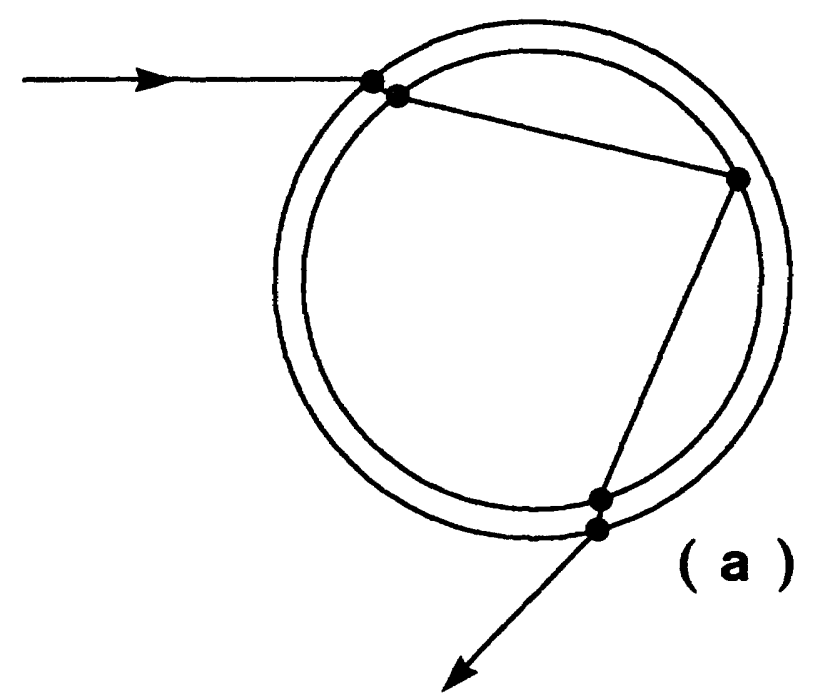

ray trajectories of Figs. 8 is described by

$$
\left.\begin{array}{c}
a_{l} \\
b_{l}
\end{array}\right\}=T_{l}^{32} R_{l}{ }^{212} R_{l}{ }^{232} R_{l}{ }^{212} T_{l}^{23} .
$$

When the core is small and the Descartes ray misses it, partial waves with $l \gg x_{12}$ reflect off the core ${ }^{23,24}$ with $R_{l}^{212} \rightarrow 1$. In Figs. 9 we show the Aden-KerkerDebye scattered intensity with the partial-wave scattering amplitudes given by Eq. (50) for $x_{23}=900, n_{1}=$ $1.333, n_{2}=1.2, n_{3}=1.0$, and for $x_{12}=600$, which corresponds to Fig. $8(\mathrm{a}), x_{12}=700$, which corresponds to Fig. 8(b), and $x_{12}=750$, which corresponds to Fig. 8(c). In ray theory, the Descartes ray touches the core when Eq. (19) is satisfied and when

$$
a_{12}=\frac{a_{23} \sin \theta_{3}{ }^{R}}{n_{2}} .
$$
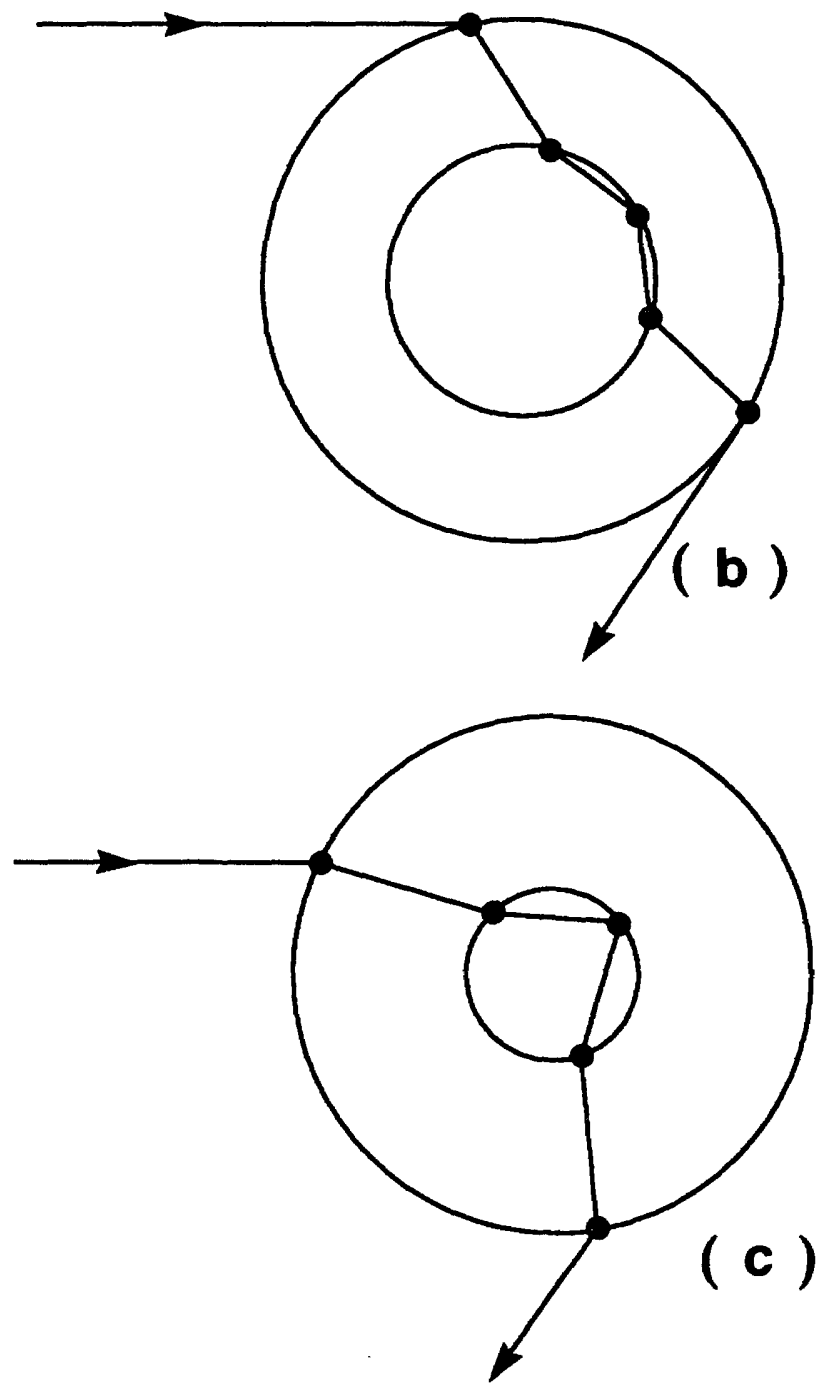

For the coated-sphere parameters of this example, the transition should occur at $x_{12}=692.82$. The results of Figs. 9 verify that in wave theory, the transition occurs for nearly the same value of $x_{12}$. Wave theory also verifies that just below the transition, one of the two supernumerary rays is blocked, greatly decreasing the amplitude of the supernumerary interference pattern while preserving the partial focusing of the rays in the immediate vicinity of the Descartes ray. The supernumerary interference pattern is not completely obliterated, however, because of interference with the surface waves created at the core-coating interface by the blocked supernumerary ray.

A more subtle transition is illustrated with ray theory in Figs. 10. Consider the $\alpha$ ray of Fig. 1(b) for the special case of $n_{1}=1.333, n_{2}=1.5$, and $n_{3}=1.0$. Let the core radius and the coating thickness be

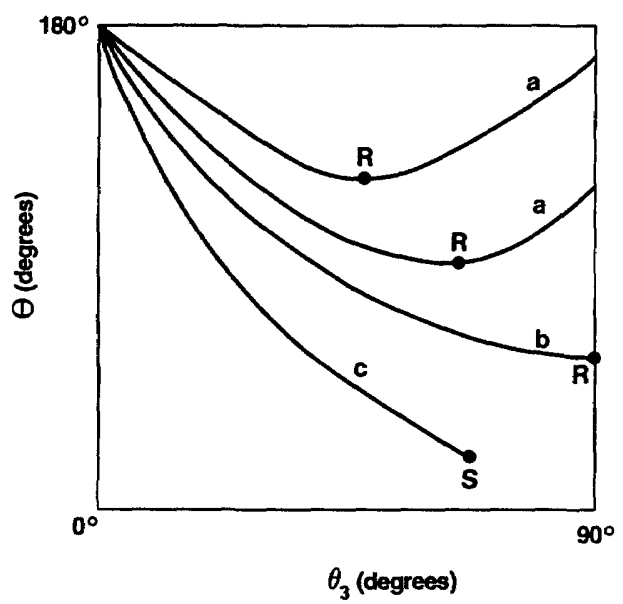

Fig. 10. Ray trajectories that correspond to the Debye-series term $T_{l}^{32} T_{l}^{21} R_{l}{ }^{11} T_{l}{ }^{12} T_{l}^{23}$ for (a) particlelike scattering for a large core, which permits rainbow formation, (b) the transition between particlelike and bubblelike scattering where rainbow extinction occurs, (c) bubblelike scattering for a small core where no rainbow can occur.

Fig. 11. Scattering angle $\theta$ as a function of the angle of incidence $\theta_{3}$ for the ray trajectories of Fig. 10. The curves labeled $a, b$, and $c$ correspond to the situations of Figs. 10(a), 10(b), and 10(c), respectively. Point $R$ denotes the rainbow, and point $S$ denotes total external reflection. The two curves labeled a correspond to two different coating thicknesses in the particlelike scattering regime. 


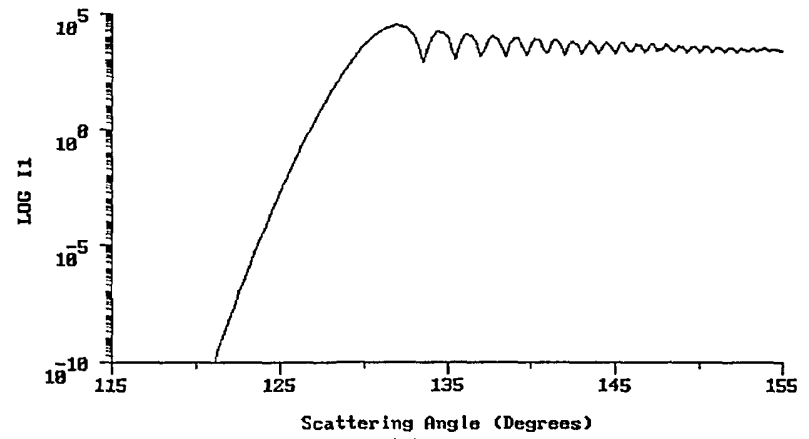

(a)

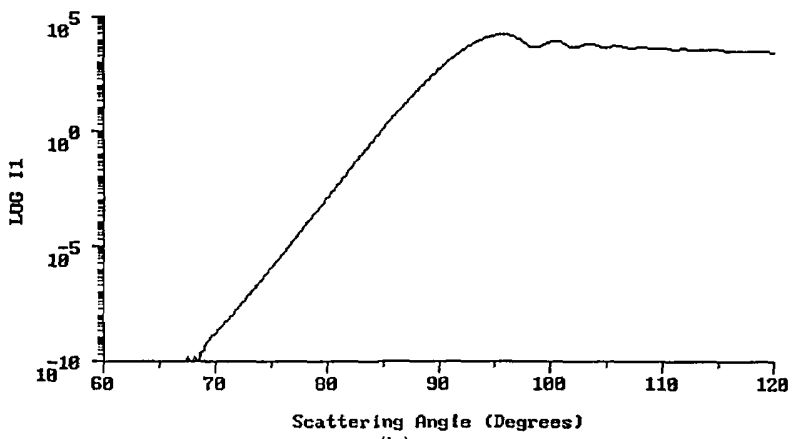

(b)

varied while the composite particle radius $a_{23}$ is held constant. If the core is large and the coating is small, as in Fig. 10(a), scattering by the coated sphere resembles scattering by a homogeneous particle that comprises core material. We saw in Section 2 that a first-order rainbow occurs for this situation. If the core shrinks in size while the coating correspondingly grows, one eventually reaches the situation of Fig. 10(c), in which scattering by the core resembles scattering by a bubble that comprises core material embedded in a large volume of coating material. For scattering by a spherical bubble, the scattering angle of the rays that make one internal reflection is a decreasing function of the impact parameter, and no first-order rainbow occurs. ${ }^{25-27}$ The behavior of the scattering angle as a function of the impact parameter was computed for a number of different coating thicknesses and is illustrated in Fig. 11. The two graphs labeled a correspond to two different coating thicknesses for the particlelike thin-coating case of Fig. 10(a), the graph labeled $b$ is the transition case of Fig. 10(b), and the graph labeled $\mathrm{c}$ is the thick-coating bubblelike case of Fig. 10(c). The point labeled $R$ is the relative minimum scattering angle that corresponds to the rainbow. As the coating thickness is increased, the angle of incidence for the rainbow also increases, reaching $\theta_{3}{ }^{R}=90^{\circ}$ at the transition. To show the migration of the relative minimum of the scattering angle clearly, two type-a curves are shown in the figure. The transition between particlelike scattering and bubblelike scattering thus occurs in ray theory when the Descartes rainbow ray is incident upon the coating-air interface at grazing incidence, and the refracted Descartes ray enters the core at

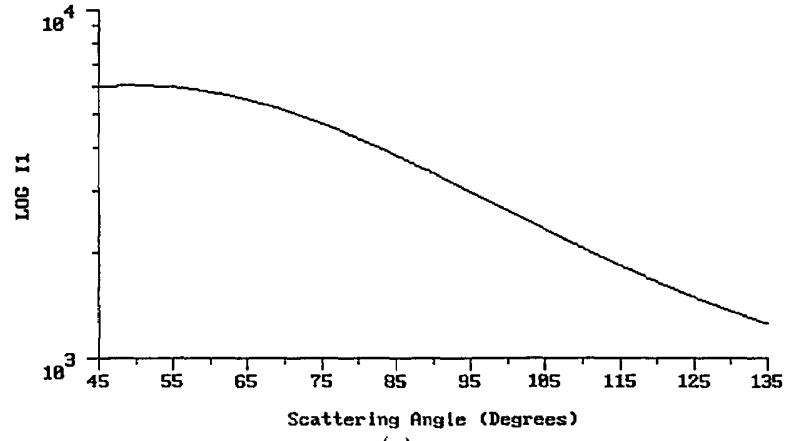

(c)

Fig. 12. Scattered intensity $\left|S_{1}(\theta)\right|^{2}$ of Eq. (54) as a function of $\theta$ for $x_{23}=900, n_{1}=1.33, n_{2}=1.5, n_{3}=1.0$ and (a) $x_{12}=850$ in the particle-like scattering regime, (b) $x_{12}=712.5$ near the rainbow extinction transition, and (c) $x_{12}=630$ in the bubblelike scattering regime.

grazing incidence with $\theta_{1}{ }^{2}=90^{\circ}$. This occurs when

$$
a_{12}=\frac{1}{n_{1}} a_{23} \text {. }
$$

As the rainbow-extinction transition occurs for grazing incidence rays, other interesting scattering effects accompany it. Because $\theta_{3}{ }^{R}=90^{\circ}$ at the transition, electromagnetic surface waves that shed radiation $^{9}$ into the angular region $\theta>\theta^{R}$ on the illuminated side of the rainbow are created at the coating-air interface. These surface waves interfere with the geometric rays that are incident with $\theta_{3}<$

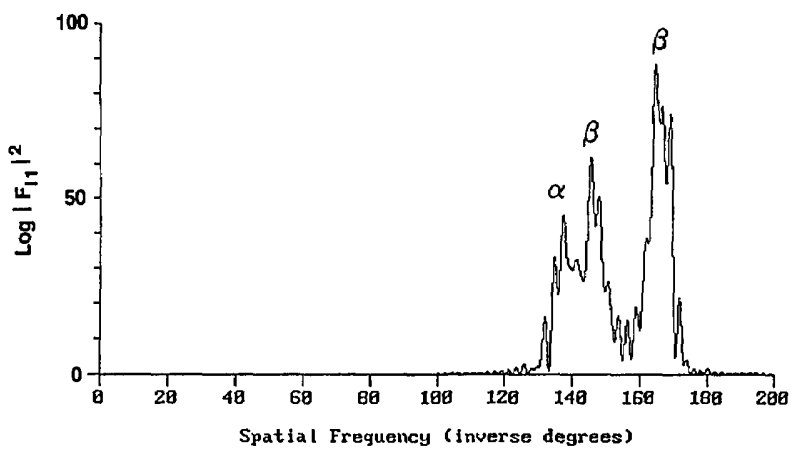

Fig. 13. Magnitude squared of the Fourier transform of the Aden-Kerker scattering amplitude $S_{\mathrm{I}}(\theta)$ of Eq. (6) as a function of spatial frequency for $x_{12}=10,000,(2 \pi \delta) / \lambda=300, n_{1}=1.333$, and $n_{2}=1.5$. The scattering amplitude was computed over a $3^{\circ}$ interval centered on $\theta=137^{\circ}$. The center of the coated sphere corresponds to $p=0$, and the outer edge corresponds to $p=174.53$ $\operatorname{deg}^{-1}$. The $\beta$ peaks are due to the $\beta$ supernumerary rays of Fig. $1(\mathrm{~b})$, and the $\alpha$ peak is due the $\alpha$ rays of Fig. $1(\mathrm{~b})$. 
$90^{\circ}$ to produce the familiar supernumerary interference pattern. This effect is novel in that for both the homogeneous sphere and for the coated sphere with

$$
\frac{a_{12}}{a_{23}}>\frac{1}{n_{1}}
$$

the supernumerary pattern is formed, at least in part, ${ }^{11}$ by the interference of geometric rays incident upon the sphere with $\theta_{3}<\theta_{3}{ }^{R}$ and geometric rays ${ }^{28}$ that are incident with $\theta_{3}>\theta_{3}{ }^{R}$. In addition, as $\theta_{1}{ }^{R}=$ $90^{\circ}$ in the core, electromagnetic surface waves are also set up at the core-coating interface. But, as $n_{1}<n_{2}$, these surface waves propagate in the direction opposite to the coating-air interface surface waves. ${ }^{9,29}$ Thus the core-coating interface surface waves shed radiation into the angular region $\theta<\theta^{R}$ on the unilluminated side of the rainbow. This is the region where normally only the complex ray contributes to the rainbow, and the scattered intensity falls off as $\exp \left[-2 / 3\left(\Theta^{R}-\theta\right)^{3 / 2}\right]$ as one progresses into Alexander's dark band. ${ }^{5}$ Because the intensity of the core-coating surface waves fall off as $\exp \left[-K\left(\Theta^{R}-\Theta\right)\right]$, where $K$ is a constant, the surfacewave mechanism should provide the dominant contribution to the intensity in Alexander's dark band.

To test these ideas, we computed the wave-theory intensity of Eqs. (4) and (6) for the Debye-series contribution to the partial-wave-scattering amplitudes:

$$
\left.\begin{array}{c}
a_{l} \\
b_{l}
\end{array}\right\}=T_{l}^{32} T_{l}^{21} R_{l}{ }^{11} T_{l}^{12} T_{l}^{23},
$$

which corresponds to the ray trajectory of Figs. 10 . The composite size parameter is $x_{23}=900$. Figure 12 (a) shows the scattering in the particlelike regime for $x_{12} / x_{23}=0.944$; Fig. 12(b) shows the scattering for $x_{12} / x_{23}=0.792$, which is just above the expected transition value of $\left(x_{12} / x_{23}\right)_{\text {transition }}=0.75$; and Fig. 12(c) shows the scattering in the bubblelike regime for $x_{12} / x_{23}=0.70$. The Aden-Kerker-Debye results confirm that the particlelike bubblelike transition occurs near the ray-theory prediction of Eq. (52). Further, in Fig. 12(b) the rainbow intensity decreases exponentially rather than faster than exponentially in Alexander's dark band, which indicates surfacewave dominance for $\Theta<\Theta^{R}$.

\section{Experimental Observations}

Perhaps the greatest difficulty in experimentally testing the results of Sections $2-5$ is the preparation of a suitable coated spherical particle. As a first attempt at an experimental confirmation, we filled an 18.4-cmdiameter thin-walled spherical glass globe with water and illuminated it with a slide projector, $10 \mathrm{~m}$ away, whose lens had been removed. A photograph of the first-order rainbow glare spots ${ }^{2,30,31}$ on the globe is shown in Plate 47. The locations of the glare spots correspond to peaks in the magnitude squared of the
Fourier transform of the scattering amplitudes. ${ }^{32,33}$ The glare spots in Plate 47 are consistent with the Fourier-transform calculation shown in Fig. 13. In addition to the usual two rainbow glare spots that are due to the $\beta$ supernumerary rays, there is a third glare spot slightly closer to the center of the globe that corresponds to the $\alpha$ ray trajectories. This multiple internal reflection effect that is due to the finite thickness of the coating is familiar in reflection off flat thin slabs of material. ${ }^{34,35}$

In summary of the results presented here, the behavior of the first-order rainbow in scattering by a homogeneous spherical particle is well understood when the wavelength, particle diameter, and particle refractive index are varied. A new and rich set of rainbow phenomena, however, occurs when the homogeneous particle acquires a coating. We have found that for three different ranges of the coating thickness, $\delta / a_{12} \lesssim 10^{-3}, \delta / a_{12} \approx 10^{-2}$, and $\delta / a_{12} \approx 0.33$, three new rainbow phenomena become important. These results reinforce our belief that the rainbow represents an incredibly fertile source of interesting optical phenomena. It is amazing that even though the rainbow has been continually studied since Theodoric of Freiberg discovered the correct ray-theory mechanism nearly 700 years ago, ${ }^{36,37}$ people are still learning new things about it.

This work was supported in part by the National Aeronautics and Space Administration grant NCC 3-204.

\section{References and Notes}

1. H. C. van de Hulst, Light Scattering by Small Particles (Dover, New York, 1981), Sec. 13.2

2. J. D. Walker, "Multiple rainbows from single drops of water and other liquids," Am. J. Phys. 44, 421-433 (1976).

3. V. Khare and H. M. Nussenzveig, "Theory of the glory," Phys. Rev. Lett. 38, 1279-1282 (1977).

4. V. Khare and H. M. Nussenzveig, "The theory of the glory," in Statistical Mechanics and Statistical Methods in Theory and Application, U. Landman, ed. (Plenum, New York, 1977), pp. $723-764$.

5. H. M. Nussenzveig, "Complex angular momentum theory of the rainbow and the glory," J. Opt. Soc. Am. 69, 1068-1079, 1193-1194 (1979).

6. D. S. Langley and M. J. Morrell, "Rainbow-enhanced forward and backward glory scattering," Appl. Opt. 30, 3459-3467 (1991).

7. A. L. Aden and M. Kerker, "Scattering of electromagnetic waves from two concentric spheres," J. Appl. Phys. 22, 1242-1246 (1951).

8. B. van der Pol and H. Bremmer, "The diffraction of electromagnetic waves from an electrical point source round a finitely conducting sphere, with applications to radiotelegraphy and the theory of the rainbow," Philos. Mag. 24, 141-176, 825864 (1937).

9. H. M. Nussenzveig, "High-frequency scattering by a transparent sphere. I. Direct reflection and transmission," J. Math. Phys. 10, 82-124 (1969).

10. J. A. Lock, "Cooperative effects among partial waves in Mie scattering," J. Opt. Soc. Am. A5, 2032-2044 (1988).

11. E. A. Hovenac and J. A. Lock, "Assessing the contributions of 
surface waves and complex rays to far-field Mie scattering by use of the Debye series," J. Opt. Soc. Am. A 9, 781-795 (1992).

12. G. Gouesbet, B. Maheu, and G. Grehan, "Light scattering from a sphere arbitrarily located in a Gaussian beam, using a Bromwich formulation," J. Opt. Soc. Am. A 5, 1427-1443 (1988).

13. J. P. Barton, D. R. Alexander, and S. A. Schaub, "Internal and near-surface electromagnetic fields for a spherical particle irradiated by a focused laser beam," J. Appl. Phys. 64, 1632-1639 (1988).

14. M. Kerker, The Scattering of Light and Other Electromagnetic Radiation (Academic, New York, 1969), Sec. 5.1.

15. C. F. Bohren and D. R. Huffman, Absorption and Scattering of Light by Small Particles (Wiley, New York, 1983), Sec. 8.1, App. B.

16. Ref. 1, Secs. 12.34 and 13.24.

17. E. Hecht, Optics, 2nd ed. (Addison-Wesley, Reading, Mass., 1987), Sec. 4.3.

18. Ref. 17, Secs. 9.41 and 9.6 .

19. G. Arfken, Mathematical Methods for Physicists (Academic, New York, 1985), Eq. (11-160) and the paragraph following it.

20. K. A. Fuller, "Scattering of light by coated spheres," Opt. Lett. 18, 257-259 (1993).

21. J. A. Lock, J. M. Jamison, and C.-Y. Lin, "Rainbow scattering by a coated sphere," in Light and Color in the Open Air, Vol. 13 of 1993 OSA Technical Digest Series (Optical Society of America, Washington, D.C., 1993), pp. 8-11.

22. R. L. Hightower and C. B. Richardson, "Resonant Mie scattering from a layered sphere," Appl. Opt. 27, 4850-4855 (1988).

23. H. M. Nussenzveig, "High-frequency scattering by a transparent sphere. II. Theory of the rainbow and the glory," J. Math. Phys. 10, 125-176 (1969), Eqs. (6.40) and (6.47).
24. J. A. Lock and E. A. Hovenac, "Diffraction of a Gaussian beam by a spherical obstacle," Am. J. Phys. 61, 698-707 (1993).

25. Ref. 23, pp. 131-132, Fig. 3a.

26. N. Fiedler-Ferrari, H. M. Nussenzveig, and W. J. Wiscombe, "Theory of near-critical-angle scattering from a curved interface," Phys. Rev. A 43, 1005-1038 (1991).

27. P. L. Marston, "Colors observed when sunlight is scattered by bubble clouds in seawater," Appl. Opt. 30, 3479-3484, 3549 (1991).

28. J. D. Walker, "Mysteries of rainbows, notably their rare supernumerary arcs," Sci. Am. 242(6), 174-184 (1980).

29. D. Ludwig, "Diffraction by a circular cavity," J. Math. Phys. 11, 1617-1630 (1970), claims that this interpretation of counterpropagating surface waves is incorrect. NumericalDebye-series computations performed by us for $p=1$ for a bubble in a homogeneous medium, however, bear out Nussenzveig's interpretation of the situation. See also Ref. 26, p. 1019.

30. J. D. Walker, "How to create and observe a dozen rainbows in a single drop of water," Sci. Am. 237(1), 138-144 (1977).

31. R. A. R. Tricker, Introduction to Meteorological Optics (Elsevier, New York, 1970), pp. 44-46.

32. J. A. Lock, "Theory of the observation made of high-order rainbows from a single water droplet," Appl. Opt. 26, 52915298 (1987).

33. H. C. van de Hulst and R. Wang, "Glare points," Appl. Opt. 30, 4755-4763 (1991).

34. Ref. 17, Figs. 4.55 and 4.60 .

35. D. S. Falk, D. R. Brill, and D. G. Stork, Seeing the Light (Harper \& Row, Cambridge, 1986), Figs. 2.42-2.43.

36. C. B. Boyer, The Rainbow from Myth to Mathematics (Princeton U. Press, Princeton, N.J., 1987), Chap. 5.

37. J. Burke, The Day the Universe Changed (Little, Brown, Boston, Mass., 1985), pp. 52-53. 